\title{
Synchronous occurrence of gastrointestinal stromal tumor and acute myeloid leukemia: A case report and review of the literature
}

\author{
NA GAO ${ }^{1,2}$, NONG-JIAN GUO ${ }^{1}$, WEN-ZHENG YU ${ }^{2}$, XUE-XIA WANG ${ }^{2}$, JIAN-RONG SUN $^{2}$, \\ NING YU ${ }^{3}$, REN-TONG LIU ${ }^{2}$, XIAO-DAN LIU ${ }^{2}$, ZENG-YAN LIU ${ }^{2}$ and RUI FENG ${ }^{2}$ \\ ${ }^{1}$ Department of Hematology, Central Hospital of Jinan, Shandong University School of Medicine, Jinan, Shandong 250013; \\ Departments of ${ }^{2}$ Hematology and ${ }^{3}$ Pathology, Binzhou Medical University Hospital, Binzhou, Shandong 256603, P.R. China
}

Received January 31, 2015; Accepted December 4, 2015

DOI: $10.3892 / \mathrm{ol} .2016 .4353$

\begin{abstract}
Gastrointestinal stromal tumors (GISTs) originate from the mesenchymal tissue of the gastrointestinal tract. The pathogenesis of GIST is associated with the mutational activation of the receptor tyrosine kinase cluster of differentiation (CD)117 or platelet-derived growth factor receptor- $\alpha$. Overall, $\sim 60 \%$ of GISTs occur in the stomach. Clinically, GISTs may coexist with various types of cancer, including liver cancer, pancreatic tumors and lymphoma, either synchronously or metachronously. The present study reports the case of a patient with the synchronous occurrence of a CD117-positive GIST and acute myeloid leukemia. A 69-year-old man was hospitalized for heart palpitations and dizziness, and was diagnosed with acute myeloid leukemia (AML) by bone marrow aspiration and flow cytometry analysis. An abdominal computed tomograpy and gastroscopy revealed the presence of GIST. The patient received chemotherapy in combination with imatinib (400 mg/day), and the mass was removed 2 months later. To the best of our knowledge, the present study is the first reported case of the synchronous development of a CD117-positive GIST and AML. Additional studies are required in order to understand the association between GIST and hematological malignancies.
\end{abstract}

\section{Introduction}

Gastrointestinal stromal tumors (GISTs) are common mesenchymal tumors of the gastrointestinal tract that are categorized as being borderline benign and malignant tumors (1). GISTs

Correspondence to: Dr Na Gao, Department of Hematology, Central Hospital of Jinan, Shandong University School of Medicine, 44 Wenhua Xi Road, Jinan, Shandong 250013, P.R. China E-mail: gn2882155@163.com

Key words: gastrointestinal stromal tumor, acute myeloid leukemia, cluster of differentiation 117, cluster of differentiation 34 arise predominantly in the stomach $(60 \%)$, small intestine (30\%) and colorectum (10\%) (2). Previous studies have elucidated that the majority of GISTs are caused by a mutation of the receptor tyrosine kinase KIT [also known as cluster of differentiation (CD)117] or platelet-derived growth factor receptor- $\alpha$ (PDGFRA) (3-5). Immunohistochemistry demonstrates that the majority of GISTs express CD117 (6). In addition, 60-80\% of GISTs diffusely express CD34 (7).

The synchronous or metachronous coexistence of GISTs and other malignancies, including liver cancer, pancreatic tumors and lymphoma, has been extensively reported (8-10). However, to the best of our knowledge, only 1 case of the synchronous development of GIST and acute myeloid leukemia (AML) has been reported in the literature (11). The present study reports the case of a patient diagnosed by GIST and AML. To the best of our knowledge, the present study is the first to report the synchronous development of a CD117-positive GIST and AML in China.

\section{Case report}

A 69-year-old man was admitted to the Binzhou Medical University Hospital (Binzhou, Shandong, China) in November 2013 due to heart palpitations, dizziness and general fatigue that had lasted for 2 months. The patient had suffered from hypertension and diabetes mellitus for $\sim 10$ years. A physical examination on admission revealed that the patient had anemia. Neither multiple superficial lymphadenopathies nor hepatosplenomegaly were evident. Preliminary investigations revealed a hemoglobin level of $76 \mathrm{~g} / \mathrm{dl}$ (normal range, $110-160 \mathrm{~g} / \mathrm{dl}$ ), platelet count of 237,000 platelets/ $\mu$ l (normal range, 100,000-300,000 platelets), white blood cell count of 32,400 cells $/ \mu \mathrm{l}$ (normal range, 4,000-10,000 cells/ $\mu \mathrm{l}$ ) and myeloblast level of $10 \%$. No abnormalities in the liver function or routine blood biochemical examination were evident. The results of the blood coagulation tests and urine analysis were not notable. The patient did not express the human immunodeficiency virus antibody.

Bone marrow aspiration revealed that blasts comprised $92.5 \%$ of the myeloid cells. The blasts were small in size and possessed pseudopodia and processes. Bone marrow biopsy 
A

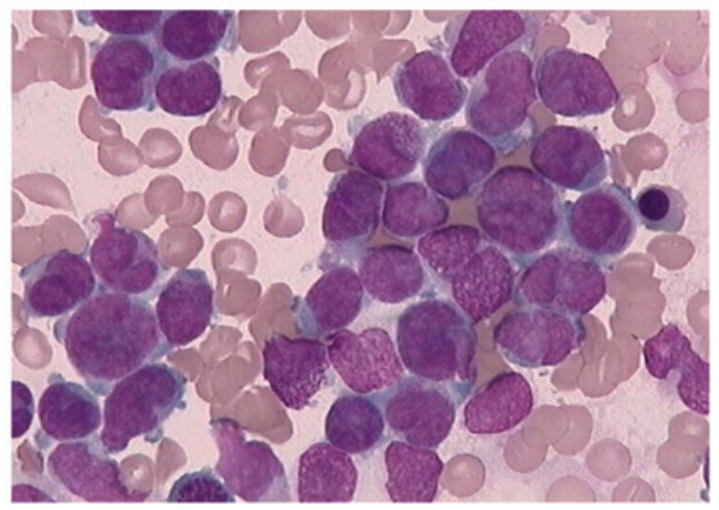

B

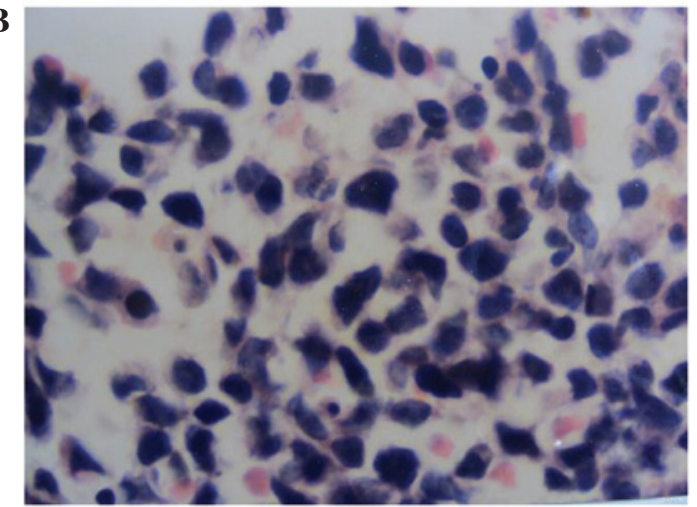

Figure 1. (A) Bone marrow aspiration (Wright-Giemsa staining; magnification, x1,000). (B) Bone marrow biopsy (hepatocyte growth factor staining; magnification, $\mathrm{x} 400)$.
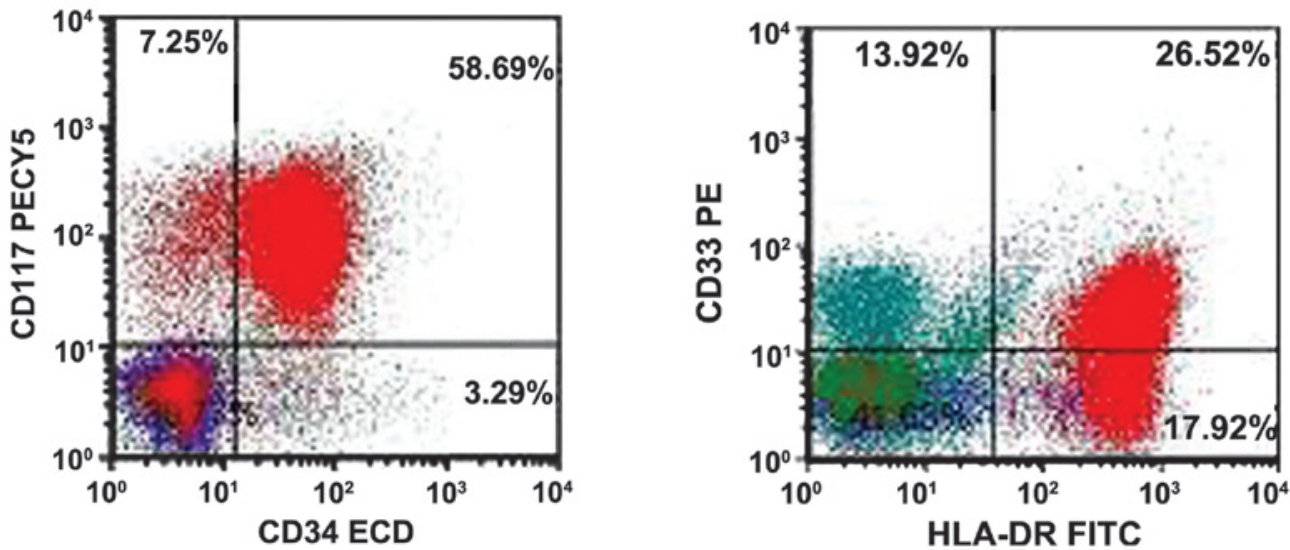

Figure 2. Flow cytometric analysis revealed that abnormal bone marrow cells expressed CD117, CD34, HLA-DR and CD33. The red signals indicate the abnormal cells. CD, cluster of differentiation; PECYS, phycoerythrin-cyanine 5; ECD, phycoerythrin-Texas Red conjugate; PE, phycoerythrin; HLA-DR, major histocompatibility complex, class II, DR; FITC, fluorescein isothiocyanate.

revealed that the patient had AML (Fig. 1). Flow cytometry analysis of the bone marrow revealed that the cellular characteristics were as follows: $\mathrm{CD} 34^{+}$; $\mathrm{CD} 117^{+}$; human leukocyte antigen-DR ${ }^{+}$; and $\mathrm{CD}_{3}{ }^{+}$(Fig. 2). Cytogenetic analysis revealed an abnormal karyotype 47,XY,+8[2]/46,XY[18] (Fig. 3). The promyelocytic leukemia (PML)/retinoic acid receptor $\alpha$ and the AML/eight-twenty one fusion gene were not expressed. From the aforementioned data, a diagnosis of AML was eventually made.

A comprehensive physical examination of the patient was conducted prior to the implementation of chemotherapy. A computed tomography scan of the abdomen revealed a lesion measuring $\sim 10 \times 9 \times 8 \mathrm{~cm}$ in size that was located in the lesser curvature of the stomach and was suspected to be a GIST (Fig. 4A). Gastroscopy revealed the presence of GISTs and bleeding in the digestive tract (Fig. 4B).

The patient received the $\mathrm{CAG}$ chemotherapy regimen (20 mg aclacinomycin, intravenous drip, days 1-4; $25 \mathrm{mg}$ cytosine arabinoside, administered subcutaneously, days 1-14; $300 \mu \mathrm{g}$ granulocyte-colony stimulating factor, administered subcutaneously, days 1-14) in combination with imatinib (400 mg, oral, days 1-14) to treat the AML, as the CAG scheme is the first choice of treatment for the elderly in China (12). The patient demonstrated complete remission following 2 courses of the Confidentiality Advisory Group scheme. In order to avoid a severe digestive tract massive hemorrhage, a subtotal gastrectomy was implemented and the patient recovered well from the procedure. The postoperative pathology suggested that the mass was a GIST lesion. The immunohistochemical staining revealed CD117, CD34 and discovered on GIST-1 (DOG1) expression, therefore also indicating that the lesion was a GIST (Fig. 5).

The patient was prescribed imatinib (400 $\mathrm{mg}$, oral, continuous) for consolidation chemotherapy, and has regularly received blood routine and liver function tests. On the basis of the follow-up results, a reduction or temporary discontinuation of the chemotherapy may be decided in the future.

\section{Discussion}

GISTs account for the majority of gastrointestinal mesenchymal tumors, which are hypothesized to originate from the interstitial cells of Cajal (13). GISTs mainly occur in older patients and there is no significant difference in the incidence of GISTs between males and females (14). The malignant risk of a GIST may be determined based on the mitotic index, size and location of the lesion (15). Previous studies indicate that certain GISTs that are small in size and possess a low 


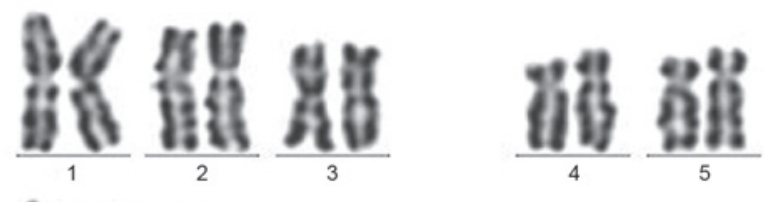

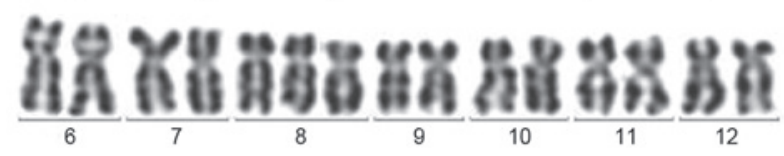

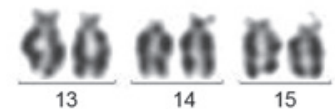

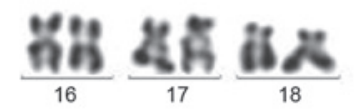

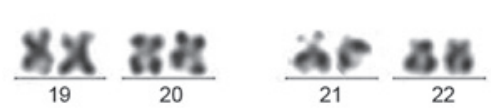

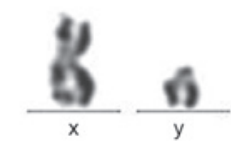

Figure 3. Cytogenetic analysis of bone marrow cells obtained from the patient revealed an abnormal karyotype of 47,XY,+8[2]/46,XY[18].

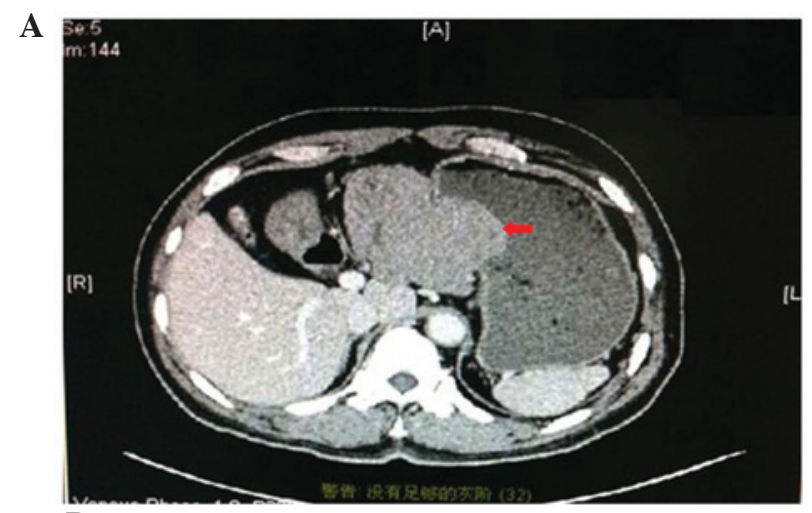

B

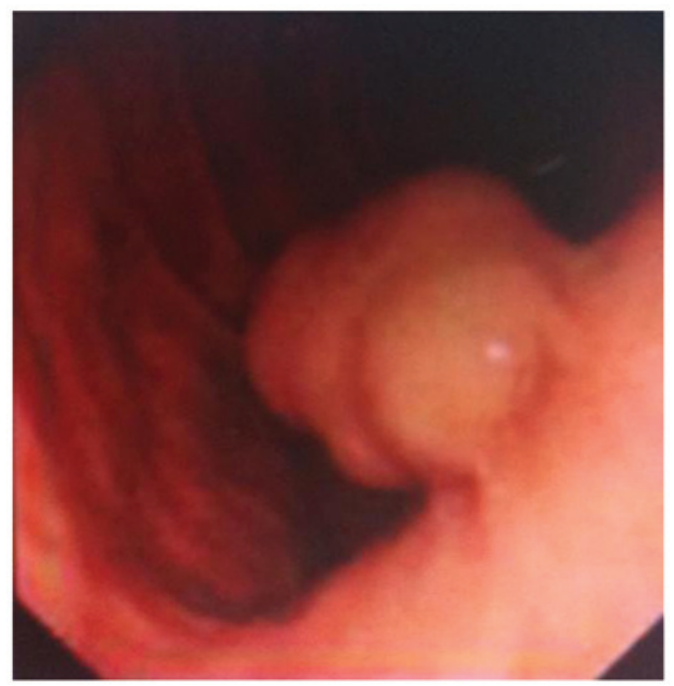

Figure 4. (A) Computed tomography revealed a giant mass (arrows) located in the lesser curvature of the stomach. (B) Macroscopic fingings of the gastroscopy revealed a giant mass.

mitotic index may adopt the features of metastasis; therefore, the concept of benign GISTs should be abandoned, as GISTs demonstrate malignant potential $(16,17)$.

The coexistence of GISTs with other malignancies has been widely reported in the literature (10). However, the synchronous occurrence of GISTs and AML has rarely been
A

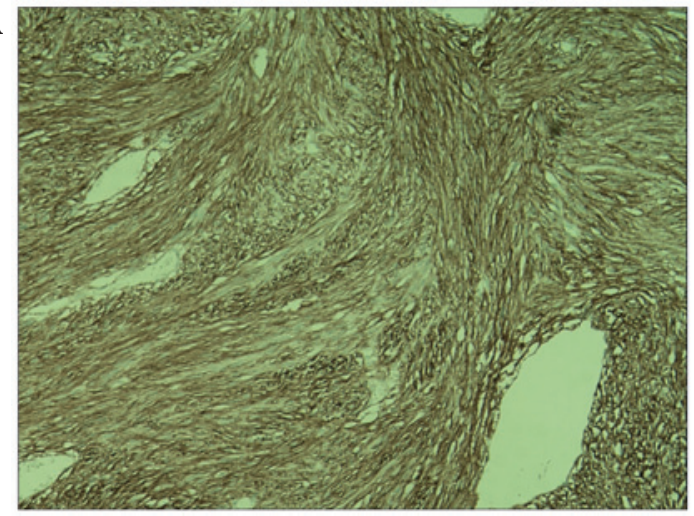

B

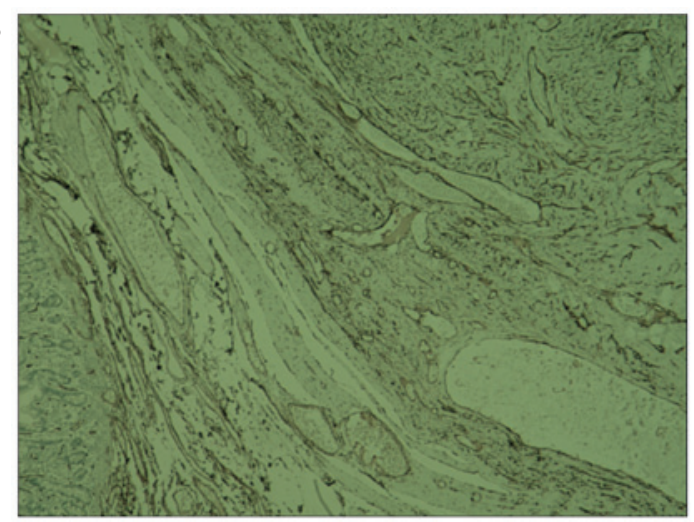

C

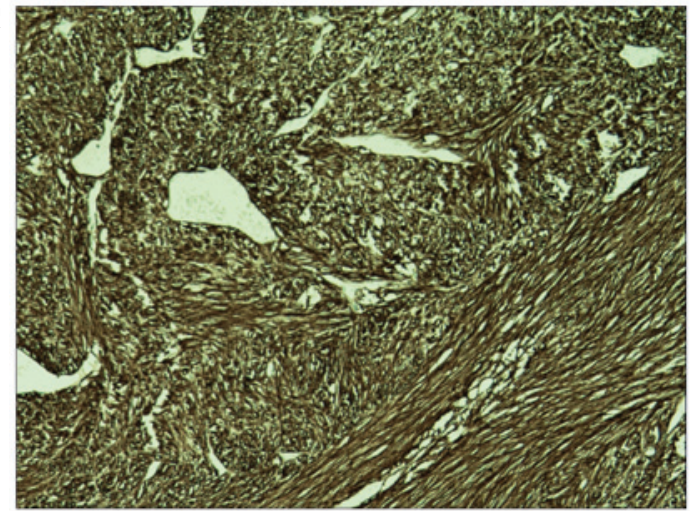

Figure 5. Immunohistochemical features revealed that the tumor expressed certain marker proteins: (A) Cluster of differentiation (CD)117, magnification, x10; (B) CD34, magnification, x4; and (C) discovered on GIST-1, magnification, $\mathrm{x} 10$.

reported. Yoshioka et al reported a case of acute PML that was diagnosed following a jejunal GIST (18). PML was considered to be the second malignancy in this study, and developed subsequent to chemotherapy or radiotherapy for GIST. In the present study, the case of a patient with synchronous occurrence of AML and GIST has been reported. To the best of our knowledge, the present study is the first to report the case of a patient that demonstrated the synchronous development of CD117-positive GIST and AML in China.

The pathogenesis of GISTs is hypothesized to be associated with the mutational activation of CD117 or PDGFRA (6). CD117, also termed KIT, has been highly conserved throughout evolution. Previous studies have indicated that the abnormal expression of genes and products caused by CD117 mutations is the major cause of GIST development $(3,19-21)$. Imatinib 
mesylate is important for the treatment of GIST, as it may alleviate symptoms, decrease post-surgery recurrence rates and prolong survival periods (1).

The immunohistochemical features of GISTs include the expression of CD117, which is widely expressed in the cytoplasm and cytomembrane of tumor cells (95\%). CD117 is widely distributed in hematopoietic cells and other tissue cells. The detection of CD117 expression is an effective method to distinguish between GISTs and other mesenchymal tumors. CD117 is recognized as a highly sensitive and specific marker for GISTs, and is also an important pathogenetic factor in AML. Similarly to GISTs, hematopoietic progenitor cells are dependent on the CD117-signaling pathway, and numerous myeloid leukemias, including AML, with $\mathrm{t}(8,21)$ and inv(16) also have CD117-activating mutations. In the present study, $t(8,21)$ was detected using conventional cytogenetic analysis. CD117 signaling is important for the regulation of red blood cell production, lymphocyte proliferation and mast cell development and function. Previous studies have demonstrated that CD117 is expressed in 68\% of patients with AML and $80 \%$ of patients with chronic myelogenous leukemia in the blast phase, but in only $2 \%$ of patients with acute lymphoid leukemia (22). Additional studies indicate that AML patients demonstrating CD117 expression have lower complete remission rates and poorer prognoses compared with AML patients that do not express CD117 (23). Common types of CD117 receptor gene mutation include exon 9 (73\%), exon 11 (10\%), exon 13 (3\%) and exon 17 (1\%) (24). CD117 receptor gene mutations in exon 17 are closely associated with a poor prognosis. Therefore, the CD117 receptor is important for AML complete remission and recurrence. Stem cell factor, the ligand for CD117, is a hematopoietic cytokine that has been detected in GIST patients, and is important for maintaining the survival of hematopoietic cells, which may lead to myeloproliferation (25), differentiation and ultimately to the occurrence of leukemia. In addition, the primitive hematopoietic tissue differentiation antigen CD34 is diffusely expressed in 60-80\% GISTs. CD117 and CD34 are important in GISTs and AML. In addition to CD117 and CD34, DOG1 is often expressed in GIST patients and is particularly important in the diagnosis of patients without CD117 expression.

The results of an epidemiological analysis in a previous study indicated a significant association between AML and GIST (26). This study indicated that AML developed 1.7-21.0 years subsequent to GIST (median interval, 6 years), and that the risk of AML was significantly higher for female patients with GIST compared to male patients with GIST. The frequency of this non-random association and the spectrum of neoplasms involved have not been sufficiently analyzed at present. The potential for a non-random and causal association between GIST and other neoplasms remains to be investigated.

\section{References}

1. Iorio N, Sawaya RA and Friedenberg FK: Review article: The biology, diagnosis and management of gastrointestinal stromal tumours. Aliment Pharmacol Ther 39: 1376-1386, 2014.

2. Chen HL, Xia JG and Ding HB: Imaging features and differential diagnosis of gastrointestinal stromal tumor. Yixue Lilun Yu Shijian 17: 2337-2339, 2013 (In Chinese).

3. Bello DM, Dematteo RP and Ariyan CE: The GIST of targeted therapy for malignant melanoma. Ann Surg Oncol 21: 2059-2067, 2014.
4. Voltaggio L, Murray R, Lasota J and Miettinen M: Gastric schwannoma: A clinicopathologic study of 51 cases and critical review of the literature. Hum Pathol 43: 650-659, 2012.

5. Corless CL, Barnett CM and Heinrich MC: Gastrointestinal stromal tumours: Origin and molecular oncology. Nat Rev Cancer 11: 865-878, 2011.

6. Miettinen M and Lasota J: Histopathology of gastrointestinal stromal tumor. J Surg Oncol 104: 865-873, 2011.

7. Fan CHL and Li ZHQ: Clinical pathological and immunohistochemical characteristics of 78 cases of gastrointestinal stromal tumor. Shi Yong Zhong Liu Za Zhi 25: 581-583, 2010 (In Chinese).

8. Liu SW, Chen GH and Hsieh PP: Collision tumor of the stomach: A case report of mixed gastrointestinal stromal tumor and adeno-carcinoma. J Clin Gastroenterol 35: 332-334, 2002.

9. Herbers AH and Keuning JJ: Staging for CLL-type non-Hodgkin lymphoma reveals a gastrointestinal stromal tumour. Neth J Med 63: 74-75, 2005.

10. Agaimy A, Wünsch PH, Sobin LH, Lasota J and Miettinen M: Occurrence of other malignancies in patients with gastro-intestinal stromal tumors. Semin Diagn Pathol 23: 120-129, 2006.

11. Joo YB, Choi SH, Kim SK, Shim B, Kim MS and Kim YJ: Synchronous development of KIT positive acute myeloid leukemia in a patient with gastrointestinal stromal tumor. Korean J Hematol 45: 66-69, 2010.

12. Hematology Society, Chinese Medical Association: Adult acute myeloid leukemia (non acute early myeloid leukemia) Chinese guidelines for diagnosis and treatment. (2011 Edition). Chinese Journal of Hematology 32: 1-4, 2011 (In Chinese).

13. Sonmez M, Arslan M, Cobanoglu U, Kavgaci H, Ozbas HM, Aydin F, Ovali E and Omay SB: Association of gastrointestinal stromal tumor and acute myeloid leukemia preceded by myelodysplastic syndrome with refractory anemia. Tumori 95 : 240-242, 2009

14. Kim MN, Kang SJ, Kim SG, Im JP, Kim JS, Jung HC and Song IS: Prediction of risk of malignancy of gastrointestinal stromal tumors by endoscopic ultrasonography. Gut Liver 7: 642-647, 2013.

15. Lewin KJ, Riddell RH and Weinstein WM $(e d s)$ : Gastrointestinal Pathology and Its Clinical Implications. Igaku-Shoin, New York, NY, pp284-341, 1992.

16. Corless CL, Fletcher JA and Heinrich MC: Biology of gastrointestinal stromal tumors. J Clin Oncol 22: 3813-3825, 2004.

17. Joensuu H: Gastrointestinal stromal tumor (GIST). Ann Oncol 7 (Suppl 10): x280-x286, 2006.

18. Yoshioka K, Yamaguchi M, Kasamatsu Y, Ashida K, Yokoh S, Tatebe A, Yoshida T and Kondo M: Acute promyoelocytic leukemia following leiomyosarcoma of the jejunum. Arch Intern Med 157: 1392-1393, 1997.

19. Antonescu CR. The GIST paradigm: Lessons for other kinase-driven cancers. J Pathol 223: 251-261, 2011.

20. Yamanoi K, Higuchi K, Kishimoto H, Nishida Y, Nakamura M, Sudoh M and Hirota S: Multiple gastrointestinal stromal tumors with novel germline c-kit gene mutation, K642T, at exon 13. Hum Pathol 45: 884-888, 2014.

21. Conca E, Miranda C, Dal Col V, Fumagalli E, Pelosi G, Mazzoni M, Fermeglia M, Laurini E, Pierotti MA, Pilotti S, et al: Are two better than one? A novel double-mutant KIT in GIST that responds to Imatinib. Mol Oncol 7: 756-762, 2013.

22. Shen HQ, Tang YM, Yang SL, Qian BQ, Song H, Shi SW and Xu WQ: Analysis of CD117 expression on leukemia cells. Zhonghua Xue Ye Xue Za Zhi 24: 228-230, 2003 (In Chinese).

23. Tsao AS, Kantarjian H, Thomas D, Giles F, Cortes J, Garcia-Manero G, Huh Y, Yang Y, Shen Y, Albitar M, et al: $\mathrm{C}$-kit receptor expression in acute leukemias-association with patient and disease characteristics and with outcome. Leuk Res 28: 373-378, 2004.

24. Wang WL, Conley A, Reynoso D, Nolden L, Lazar AJ, George S and Trent JC: Mechanisms of resistance to imatinib and sunitinib in gastrointestinal stromal tumor. Cancer Chemother Pharmacol 67 (Suppl 1): S15-S24, 2011.

25. Chen SQ and Xiong AQ: The progress and implication of stem cell factor. Basic Med Sci Clin 22: 385-390, 2002.

26. Miettinen M, Kraszewska E, Sobin LH nd Lasota J: A nonrandom association between gastrointestinal stromal tumors and myeloid leukemia. Cancer 112: 645-649, 2008. 